\title{
Design of a UWB Printed G-shaped Monopole Antenna using Characteristic Modes
}

\author{
Farhad Gozasht, Kevin Po and Ananda Sanagavarapu Mohan \\ Centre for Health Technologies, Faculty of Engineering and IT, \\ University of Technology Sydney (UTS), Sydney, Australia. \\ Email: Farhadgozasht@student.uts.edu.au, Ananda.Sanagavarapu@uts.edu.au
}

\begin{abstract}
This paper presents a novel G-shaped compact monopole UWB antenna printed on FR4 substrate with coplanar waveguide (CPW) feeding. The proposed antenna operates over the frequency band between 3 and $11 \mathrm{GHz}$ offering a fractional impedance bandwidth of around $110 \%$. The antenna is designed by analysing the modal currents calculated using the characteristic mode theory. Experimental results on reflection coefficient agree well with simulations and the proposed antenna has a desirable gain and radiation pattern characteristics over the ultra-wideband frequency range. The overall dimensions of the antenna are $28 \times 35 \times 1.6 \mathrm{~mm}^{3}$ which makes it a good candidate for many ultra-wide-band radio applications.
\end{abstract}

Keywords-ultra wideband (UWB), printed monopole antenna, characteristic modes, $\mathrm{CPW}$ feed.

\section{INTRODUCTION}

The ultra-wideband (UWB) radio technology is finding applications in high data rate wireless communication systems, cognitive radio, radars, biomedical imaging, indoor localisation and tracking etc. [1-2]. Although the antenna design considerations are usually dictated by the specific application and system considerations, in general, compact sized planar antennas having reflection coefficient, gain, radiation pattern and linear phase response etc. over extremely wide bandwidth are preferred. It has been well established that planar monopole UWB antennas offer appealing features such as simple geometry, very wide bandwidth over 3 to $11 \mathrm{GHz}$, compact in size, omnidirectional radiation and ease of manufacturing [14].

In view of these interesting characteristics, a number of planar monopoles with various optimised geometries have been investigated for UWB extensively [3]-[6], [10]. The most commonly used feeding for printed monopoles is the coplanar wave guide feeding. Also, a variety of printed slot antennas are available, e.g., a monopole-like slot antenna with CPW feeding for UWB applications [14].

Although a number of printed monopole antennas with optimised performance are published in the literature, but a very few analyses characterising the physical behaviour of these type of antennas are available. The Theory of Characteristic Modes (TCM) originally proposed by Garbacz [8] and later refined by Harrington [9] analyses the modal behaviour of antennas in order to provide more physical interpretation of the radiation phenomena taking place on the structure. TCM has also been used successfully for the design and analysis of various wire and planar antennas [12, 13]. In this paper TCM is used to provide physical insight into the radiating behaviour of a printed UWB planar monopole antenna, with an aim for performance improvement over a wideband of frequencies. In following sections, it will be shown as to how the information provided by the modal analysis would help with the design of the antenna.

In this paper, we propose a planar, CPW fed printed Gshaped monopole antenna and attempt to design it using the theory of characteristic modes to achieve operation over the UWB band. To start with, we consider a printed CPW-fed square monopole antenna structure and analyse its performance using the characteristic mode theory which has led to the modification of its shape to obtain a G-shaped monopole to finally achieve our objectives. The designed G-shaped antenna has a compact size of $28 \times 35 \times 1.6 \mathrm{~mm}^{3}$ and offers a fractional impedance bandwidth of $114 \%$ with a radiation pattern that is relatively uniform over the band. We use FEKO [7] for antenna and characteristic mode analysis and compare the calculated data with measured results on impedance bandwidth.

\section{ANTENNA CONFIGURATION}

A square monopole antenna printed on a FR4 substrate with CPW feeding was first chosen and the antenna shape modified by symmetrically bevelling at its lower side for bandwidth improvement [10]. Firstly, the impedance characteristics for different planar geometries were calculated, and then the characteristic modes were obtained with the aim of enforcing the total surface current to flow over the whole surface of the radiator as it needs to operate over a wide frequency band. After a number of iterations, we obtain the proposed G-shaped monopole antenna with CPW feeding. The antenna has a single metallic layer and is printed on one side of a FR4 substrate whose thickness is $1.6 \mathrm{~mm}$; the dielectric constant is 4.7 and 4.7 and the loss tangent of 0.0038 .

\section{A. Theory of characteristic modes (TCM)}

Characteristic modes are real current modes that can be computed numerically for both conducting and dielectric bodies of arbitrary shape [8]-[9], [13]. Characteristic modal currents $J_{n}$ can be defined as a set of orthogonal real surface currents which depend on object shape and size, and dielectric properties but are independent of any excitation source. The total current distribution on the antenna is the superposition of these characteristic modes with appropriate weighting 
coefficients. Since characteristic modes form a set of orthogonal functions, they can be used to expand the total current on the surface of the body [9]. By definition, the modes are related to the power that can be radiated by the body. Associated to the characteristic currents, a set of characteristic fields $E_{n}$ can be computed. Therefore, the field radiated by the antenna can be expressed as a superposition of these characteristic fields or modal fields. The characteristic modal currents can be obtained as the eigen functions of the following particular weighted eigenvalue equation [9], [13] as

$$
X\left(\vec{J}_{n}\right)=\lambda_{n} R\left(\vec{J}_{n}\right)
$$

where $\lambda_{\mathrm{n}}$ are the eigenvalues, $\vec{J}_{n}$ are the eigen currents, and $R$ and $X$ are the real and imaginary parts a of the impedance matrix $\mathrm{Z}$ calculated by solving the associated integral equation using the Method of Moments. Each characteristic mode is associated with an eigenvalue, $\lambda_{\mathrm{n}}$. This parameter provides useful insight: its magnitude provides information on the radiation of the associated modes and the reactive power of a mode is also proportional to the magnitude of its eigenvalue. In other words, a particular mode whose eigenvalue has smaller magnitude, can contribute to the antenna radiation when excited [13]. Furthermore the sign of the eigenvalue determines whether the mode contributes to storing of magnetic energy $\left(\lambda_{n}\right.$ $>0)$ or electric energy $\left(\lambda_{n}<0\right)$.

Usually, a quantity known as 'modal significance' $\left(M S_{n}\right)$ [11] is commonly used for antenna characterisation and it represents the normalized amplitude of the current modes,

$$
M S_{n}=\left|\frac{1}{1+j \lambda_{n}}\right|
$$

$\mathrm{MS}_{\mathrm{n}}$ determines as to how much each mode contributes to the total radiation. The resonance for each mode occurs ideally when $\lambda_{\mathrm{n}}=0$ at which modal significance has the maximum value of unity.

\section{A. Antenna Analysis}

Figure 1 shows the printed bevelled square monopole antenna on FR4 substrate whose geometrical parameters are tabulated in Table I. Figure 2 shows the calculated reflection coefficient for this antenna geometry obtained using FEKO $^{\mathrm{TM}}$ which shows that the antenna does not resonate at frequencies below $5 \mathrm{GHz}$ and has multiple narrowband resonances above $5 \mathrm{GHz}$. This is confirmed by obtaining the modal significance (MS) as shown in Fig 3 which is also obtained using FEKO ${ }^{\mathrm{TM}}$ [7]. We have chosen six characteristic modes for this antenna. From figure 3 , one can observe that at frequencies around 5 $\mathrm{GHz}$, just first two modes have a value of MS closer to unity which mean that these are the main radiating modes at this frequency and above. The MS values of the remaining four modes $j_{3}-j_{6}$ tend to unity at around $7 \mathrm{GHz}$ at which they may contribute to the antenna radiation.

We have also investigated the characteristic current modes on the antenna shown in Fig. 1 by calculating the eigenvalues of the first six current modes $J_{l-6}$.

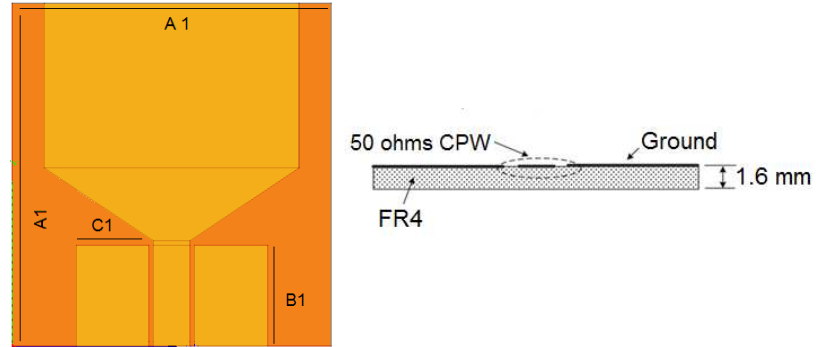

Fig. 1. Geometry of CPW fed square monopole with symmetric beveling.

TABLE I. Antenna Dimensions of SQuare Monopole

\begin{tabular}{|l|c|c|c|}
\hline \multirow{2}{*}{$\begin{array}{c}\text { Antenna } \\
\text { size }\end{array}$} & \multicolumn{3}{|c|}{ Antenna's Dimensions in mm } \\
\cline { 2 - 4 } & $\boldsymbol{A 1}$ & $\boldsymbol{B} \boldsymbol{1}$ & $\boldsymbol{C} \boldsymbol{1}$ \\
\hline $\mathrm{mm}$ & 51 & 18 & 14 \\
\hline
\end{tabular}

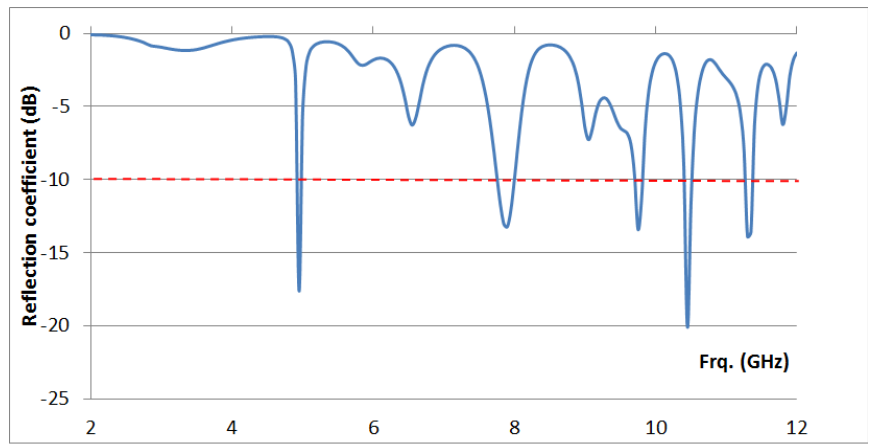

Fig. 2. Reflection coefficient for antenna in Fig.1

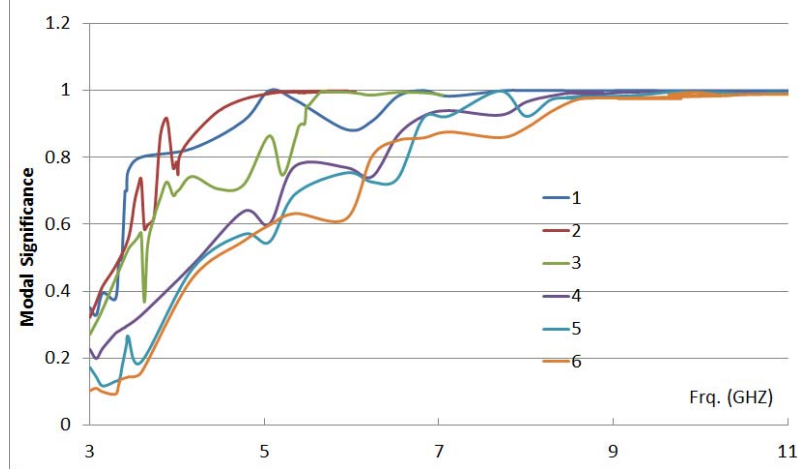

Fig. 3. Modal significance for antenna in Fig.1

a)
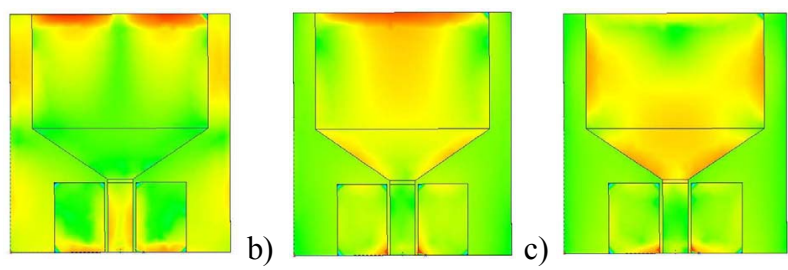

Fig. 4. First three modal currents at $5 \mathrm{GHz}$ for antenna shown in Fig.1. 
Then, the modal currents are computed to investigate the contribution of characteristic modes to the antenna radiation at given frequencies. Since the total current is formed by the contribution of a set of orthogonal current modes that have a defined bandwidth, by exciting as many modes as possible may enhance the total bandwidth. Fig 4 shows that for the antenna in Fig.1, at $5 \mathrm{GHz}$ the first three modal currents do not flow over the whole surface of the radiator and hence its radiation performance is inferior. To improve the radiation performance over ultra-wideband, we will now modify the antenna geometry to force these modal currents to flow over the whole surface of the antenna. Fig. 5 shows the G-shaped monopole antenna which is obtained by modifying the geometry shown in Fig.1. The geometrical dimensions of the G-shaped monopole antenna are tabulated in Table II.

Figure 6 shows the MS of the G-shaped antenna in which we can observe that from $3 \mathrm{GHz}$ the first three modes have an MS close to unity meaning that they contribute to radiation around $3 \mathrm{GHz}$ and above. Thus, the modified structure in Fig.5 can excite all these modes even for frequencies below $5 \mathrm{GHz}$. Fig.7 shows that the first four modal currents flow over the whole surface of the radiating patch for the G-shaped antenna. This probably would have caused the uniform omnidirectional radiation as shown in Figure 10.

To further reduce the size of the antenna, empirical optimization of antenna geometry is carried out using $\mathrm{FEKO}^{\mathrm{TM}}$. As the size of the antenna changes, the current modes also cause changes to the input impedance [5].

We also modified the G-shaped monopole configuration by introducing two pairs of symmetrical notches having a width of $0.5 \mathrm{~mm}$ and a length of $1 \mathrm{~mm}$ in the feed line to obtain a slightly better reflection coefficient for the lower end of the band without affecting the VSWR at higher frequencies. The appropriate feeding position has been obtained by performing numerical experiments using FEKO $^{\mathrm{TM}}$ [7]. Thus final UWB performance of the proposed compact size antenna is achieved using an approach that combines TCM with empirical parametric optimisation.

\section{RESULTS AND DISCUSSION}

The fabricated antenna was tested using an HP 8720A Vector Network Analyser. Figure 8 shows the measured and simulated $\mathrm{S}_{11}$ curves. Figure 8 also shows the simulated $\mathrm{S}_{11}$ curves by varying the slot angle (B) while other antenna parameters are fixed. It can be seen from this figure when B is smaller than 22 degrees, the antenna does not resonate at the frequency band over $7 \mathrm{GHz}$. Fig. 9 shows calculated gain of the proposed G-shaped monopole antenna .The calculated 3-D radiation patterns of antenna shown in Fig.5(a) at 4,5 and 7 $\mathrm{GHz}$ are shown in Fig. 10.

The finalized antenna structure has a volume of about $1.6 \mathrm{~cm}^{3}$, which is quite compact for many UWB wireless applications. The simulated and measured performance of the antenna shows that antenna can cover a wide band from 3 to 11 $\mathrm{GHz}$ with an omnidirectional radiation pattern.
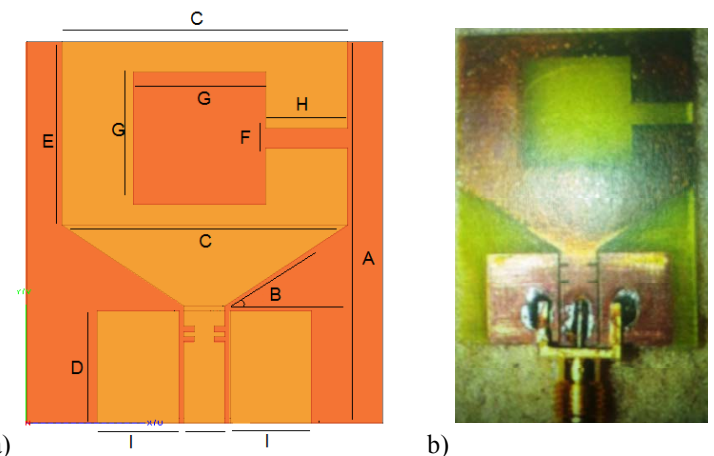

b)

Fig. 5. a) Geometry of UWB G-shaped CPW fed Monopole-like antenna. b) The fabricated G-shaped CPW fed Monopole antenna.

TABLE II. DIMENSIONS FOR G-SHAPED MONOPOLE

\begin{tabular}{|c|c|c|c|c|c|c|c|c|c|}
\hline \multirow{2}{*}{$\begin{array}{c}\text { Antenna } \\
\text { size }\end{array}$} & \multicolumn{10}{|c|}{ Antenna's Dimensions in mm } \\
\cline { 2 - 10 } & $\boldsymbol{A}$ & $\boldsymbol{C}$ & $\boldsymbol{D}$ & $\boldsymbol{E}$ & $\boldsymbol{F}$ & $\boldsymbol{G}$ & $\boldsymbol{H}$ & $\boldsymbol{I}$ & $\boldsymbol{B}$ \\
\hline Milimiters & 35 & 28 & 11 & 18 & 2 & 13 & 8 & 8 & $\begin{array}{c}33 \\
\text { Degrees }\end{array}$ \\
\hline
\end{tabular}

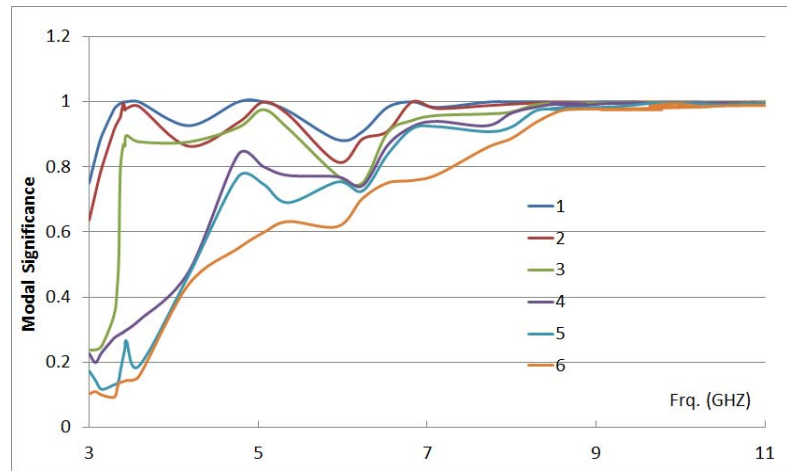

Fig. 6. Modal significance check mode 123 at 6 a)
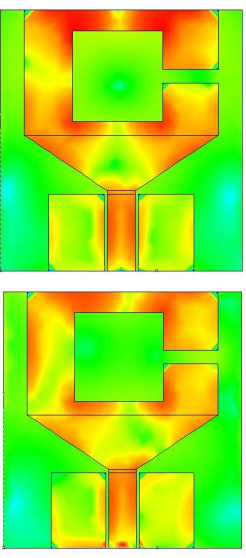

b)

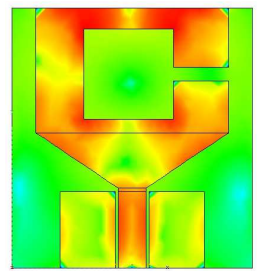

d)

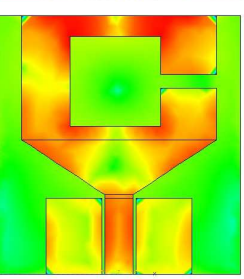

Fig. 7. First four modal currents at 5GHz for antenna shown in Fig.5(a). 


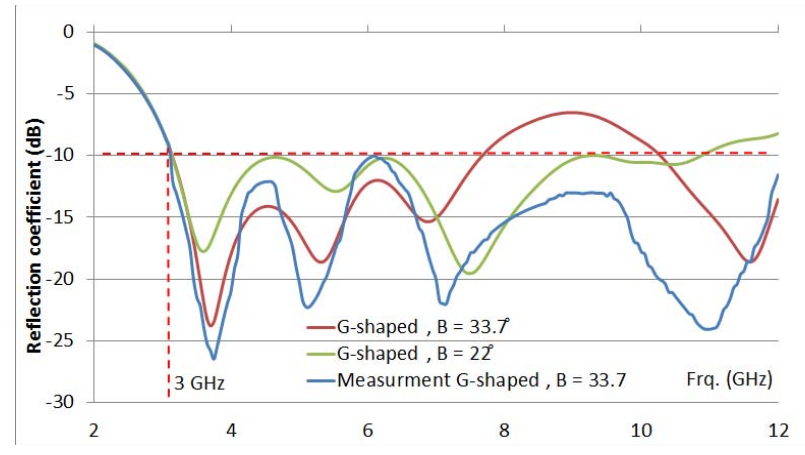

Fig. 8. Reflection Coefficient versus frequency for G-shaped monopole antenna.

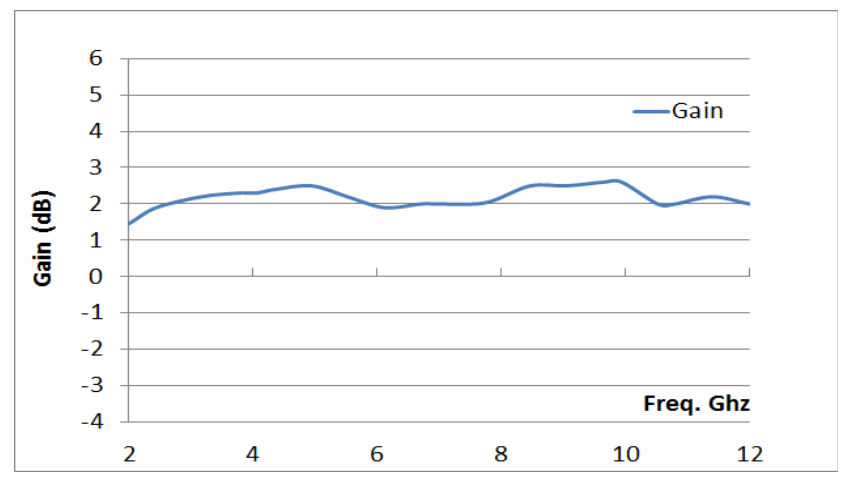

Fig. 9. Calculated Max. Gain of the antenna shown in Fig.5(a).
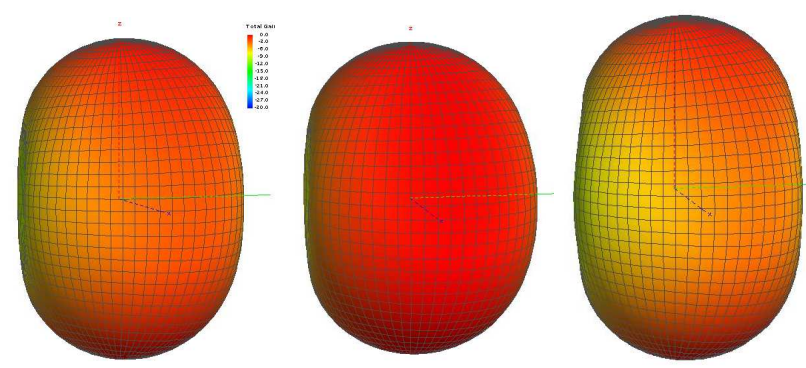

Fig. 10. Calculated 3-D radiation pattern at 4, 7, $9 \mathrm{GHz}$ for antenna shown in Fig.5(a).

\section{CONCLUSION}

A novel and compact UWB G-shaped printed monopole antenna with $\mathrm{CPW}$ feeding is proposed. The theory of characteristic modes is used to analyse and optimize the radiation behaviour of the antenna. The measured reflection coefficient of the proposed antenna has an acceptable agreement with simulations over the chosen band.

\section{REFERENCES}

[1] H. Schantz, "A brief history ofUWB antennas," IEEE Aerosp. Electron. Syst. Mag., vol. 19, no. 4, pp. 22-26, 2004.

[2] Wu, Q., Jin, R., Geng, J., Ding, M.: 'Printed omni-directional UWB monopole antenna with very compact size', IEEE Trans. Antennas Propag., 2008, 56, pp. 896-899

[3] S. Y. Suh, W. L. Stutzman, and W. A. Davis, "A new ultrawideband printed monopole antenna: the planar inverted cone antenna (PICA)," IEEE Trans. Antennas Propag., vol. 52, no. 5, pp. 1361-1364, May 2004.

[4] A. J. Kerkhoff, R. L. Rogers, and H. Ling, "Design and analysis of planar monopole antennas using a genetic algorithm approach," IEEE Trans. Antennas Propag., vol. 2, pp. 1768-1771, Jun. 2004.

[5] C.-W. Ling and S.-J. Chung, "A simple printed ultrawideband antenna with a quasi-transmission line section," IEEE Trans. Antennas Propag., vol. 57, no. 10, pp. 3333-3336, 2009.

[6] S. T. See and Z. N. Chen, "An Ultrawideband Diversity Antenna" IEEE Trans. Antennas Propag., vol. 57, no. 6, pp. 1597-1605, 2009.

[7] $\mathrm{FEKO}^{\mathrm{TM}}$, EM Software \& Systems, http://www.feko.info

[8] R. Garbacz and R. Turpin, "A generalized expansion for radiated and scattered fields," IEEE Transactions on Antennas and Propagation, vol. 19, no. 3, pp. 348 - 358, May 1971.

[9] R. Harrington and J. Mautz, "Theory of characteristic modes for conducting bodies," IEEE Transactions on Antennas and Propagation, vol. 19 , no. 5, pp. $622-628$, Sep. 1971.

[10] X.N. Qiu, H.M. Chiu and A.S. Mohan, "Symmetrically beveled Ultra Wideband Planar Monopole Antenna" IEEE Antennas and Propagation Society International Symposium Digest, vol. 2A, pp. 504 - 507, July, 2005.

[11] B. A. Austin and K. P. Murray, "The Application of CharacteristicMode Techniques to Vehicle-Mounted NVIS Antennas," IEEE Trans. Antennas Propagat., vol. 40, no. 1, pp. 7-21, Feb. 1998.

[12] Y. Lu, Y. Huang, H.T. Chattha and Y. Shen, "Technique for minimising the effects of ground plane on planar ultra-wideband monopole antennas", IET Microw. Antennas Propag., Vol. 6, no. 5, pp. 510-518, 2012.

[13] M. Cabedo-Fabres, E. Antonino-Daviu, A. Valero-Nogueira and M Ferrando-Bataller, "The Theory of Characteristic Modes Revisited: A Contribution to the Design of Antennas for Modern Applications", IEEE Antennas and Propagation Magazine, Vol. 49, no. 5, pp.52 - 68, Oct. 2007.

[14] X. Qing Z.N. Chen ,"Compact coplanar waveguide-fed ultra-wideband monopole-like slot antenna", IET Microw. Antennas Propag., Vol. 3, Iss. 5, pp. 889-898, 2009. 